\title{
The Landscaping Art and Cultural Interpretation of Walls in Traditional Suzhou Buildings
}

\begin{abstract}
Shu Yang ${ }^{1, *}$ Lin Gui $^{1}$
${ }^{1}$ Suzhou Institute of Trade \& Commerce, Suzhou, Jiangsu 215122, China

*Corresponding author. Email: dhlg2011@163.com

ABSTRACT

This paper takes the most basic and most important physical element walls among the architectural elements as the main body of the research, takes traditional Suzhou buildings as the background, mainly takes the space spirit, form characteristics, cultural connotation and aesthetic characteristics of Suzhou traditional architecture as the perspective, and uses the method of combining diachronism and synchronicity to study the landscaping techniques and cultural interpretation of the walls in traditional Suzhou buildings (mainly Suzhou traditional residential buildings and Classical Gardens of Suzhou). As far as the wall landscaping technique is concerned, the patterns of traditional Suzhou buildings blend the aesthetic characteristics of China and the West, and construct a new aesthetic system, which is manifested in the aesthetic construction of form and content, and the emotional blending of subject and object, and finally through the situation construction related to social culture, the landscaping aesthetics of the walls of traditional Suzhou buildings are integrated into the interpretation of cultural connotations.
\end{abstract}

Keywords: Suzhou, walls, landscaping, cultural interpretation

\section{INTRODUCTION}

Among the architectural elements, the wall is the most basic and most important physical element, and it is the carrier of the architectural spirit. The traditional Suzhou buildings have an important position in the history of Chinese architecture, and their walls are naturally unique. Today's architecture and landscape design draw more and more lessons from Suzhou's traditional elements. How to design modern architecture and landscape design with traditional Suzhou characteristics has attracted more and more attention. This requires a deep understanding of the spatial spirit, form characteristics and cultural connotations of the walls in traditional Suzhou buildings, rather than simply imitating and copying traditional elements. From the perspective of the interactive relationship between walls and space, this paper explores the landscaping techniques and cultural interpretation of walls in traditional Suzhou buildings, and tries to further understand the essence of traditional Suzhou buildings, so as to provide reference and inspire modern architecture and landscape design.

\section{WALLS IN TRADITIONAL RESIDENTIAL BUILDINGS IN SUZHOU}

\section{A. Aesthetics of form}

Ordinary traditional residential buildings in Suzhou are the largest number of traditional buildings in Suzhou. The water network in Suzhou is dense, and the relationship between Suzhou residential buildings and river water is very close. Suzhou residential buildings can be roughly divided into two categories: one type is the residential buildings along the river close to the river, and the other type is the a mansion with many courtyards and very high walls far from the river. The walls of these two types of residential buildings have the common characteristics of white walls and black tiles, but also have their own formal beauty.

The masonry of the walls of the residential buildings along the river in Suzhou is mostly connected by a mixture of various materials. The part close to the ground and water is built with stone, mainly for waterproof and moisture-proof. The walls of the residential buildings along the river undulate along the revetment, concaving in and protruding out, and cleverly forming a simple and elegant exterior wall landscape. The modeling of walls of the residential buildings along the river on Shantang Street in Suzhou are light and concise, with light and elegant colors, smooth lines, and soft and clear outlines. The 
architectural art features of clarity, elegance, lightness, and simplicity are reflected everywhere, which are permeated with thick residential culture breath.

Suzhou people are restrained in character, and the courtyards and residential buildings built deep in the alleys are almost built with "high walls with bricks". The courtyard walls of ancient residential buildings are solid, thick and solid, and the inner walls are mostly continuous and interlinked. In addition, the doors and windows around the hall and Saikan in the courtyard wall are all transparent and hollow, which are in sharp contrast with the outward closed enclosure. The alleys with high walls are mostly straight narrow alleys. Although it is high wall and narrow alley, the gables on both sides are naturally staggered along the alley, with a abstinent degree of stretching and retracting. Most of the indoor rooms of the house also use white walls to make it full of light. Because the old-style wooden carved lattice windows have weak lighting quantity, the reflection of the white wall plays a role. The use of white walls in the interior will not affect its beauty, on the contrary, it can bring out all the interior layouts. Chinese classical furniture has a darker wood color, which is contrasted by the light-colored wall behind, making the figure clear and prominent.

The deep courtyard is separated by scattered white walls. The tops of some walls are densely arranged with black tiles, which is extraordinary; some walls even fall into a stepped style, which are called "screen walls", which are extremely rich in layering. There are various types of ornamental perforated windows on some walls, fascinating people to explore between the hidden and the obvious. The high wall is closed, which looks a bit depressing, but it leaves a wonderful space: the high white walls in a mansion with spacious halls and extensive gardens are tightly enclosed to form a patio. The patio is not only responsible for the lighting and ventilation of the room, but also reveals an attitude of life and a cultural atmosphere.

\section{B. Functional suitability}

The summer in Suzhou is hot, rainy and humid, so it is an important functional requirement for the walls of Suzhou residential buildings to escape the heat and moisture. This requires buildings to have good ventilation and at the same time avoid direct sunlight. In order to create such a suitable living environment, Suzhou people use a large number of architectural components such as grilles and ornamental perforated windows to combine with brick and stone walls to form a transparent space. Under the premise of satisfying the basic functional requirements such as lighting and ventilation, the wall enclosing the building can connect the indoor and outdoor spaces to the greatest extent through appropriate hollow parts to prevent the space from being completely isolated. The use of window sashes, grilles, bamboo curtains, etc. can bring vision penetration, but will not completely block light and shadow, which is beneficial to daily life. At the same time, this kind of hollow construction forms a space that is different from a completely physical enclosure, allowing the penetration of the line of sight and forming a faintly discernible rich feeling of the extended space. In order to improve the heat in summer, patios are set up in the front and back of the lobby and balcony. Generally speaking, the front patio is larger, and the depth is equal to the eaves height of the hall to facilitate ventilation and sunshine. The back patio is small and narrow, commonly known as the "crab eye patio". The small patio faces the high boundary wall to the north, which can avoid direct sunlight in summer and prevent a western exposure. The open north window of the hall in summer can also guide the airflow along the high wall to generate a whirlwind and obtain good convective ventilation. In winter, it can resist the invasion of northwest wind. Due to the small depth, the sunlight reflected on the white wall can increase the brightness of the room.

\section{WALlS IN Classical GaRdens OF SUZHOU}

There are various types of walls in Classical Gardens of Suzhou. According to the material and structure of the "wall", the walls are divided into four types in "Art of Garden-building": whitening wall, ground brick wall, leaky brick wall and random stone wall. In the form of expression, the garden wall stresses that "many aesthetic interests should be expressed in an implicit way, rather than too obvious", pursuing the depth of artistic conception. The space performance emphasizes that "It will be too deep if it's separated, and it will be too shallow if it's broken through". In these landscaping and expression techniques, the garden wall plays an extremely important role.

\section{A. Innovative landscaping techniques}

1) Obstructive scenery: When borrowing sceneries in Classical Gardens of Suzhou, in order to realize that "if the outside scenery is vulgar, try to cover it; if it is beautiful, try to borrow it to use", it often uses different garden walls to cover the "vulgar" part of the outside scenery. In order to conceal the inferiority and hide the inadequacy, hide first if it wants to present, and create a garden artistic conception that "a small path opens up on an enchanting view" and "deep courtyard", different garden walls are often used to obstruct the scenery. Obstructive scenery mainly has the functions of stimulating aesthetic appeal, showing the beauty by obstructing the ugly, increasing the level of the garden, and enriching the garden landscape. The most common thing in Classical Gardens of Suzhou is to set up a 
courtyard wall at the entrance of the garden design, so as to achieve the effects of compliment after criticism, increasing the level, organizing the flow of people, and showing the beauty by obstructing the ugly. In addition, a garden wall is set at the end of the landscape sequence as a barrier to make visitors linger around.

2) Scene that serves as a foil: If whitening walls are used in the garden without exception, it will inevitably appear monotonous, so whitening walls in gardens often use the method of scene that serves as a foil. The whitening walls have a smooth texture, just like drawing paper. For example, the scenery wall of Haitang Chunwu in The Humble Administrator's Garden in Suzhou uses white walls as paper and bamboo, wood, flowers and stacked stones to create a vivid main landscape; for another example, in order to express the "main peak" hillstone in the courtyard, the Wufengxian Pavilion in the Lingering Garden uses a method of diminishing the architectural image. The back of the hillstone is set off with a white wall. The entire space is simple and lively with a prominent theme.

3) Leaking scenes and showing scenes: The walls of the garden often have door openings and hollow windows, and their styles are very rich. Door openings and hollow windows are generally framed with wet sanding bricks, with moldings polished on the edges. Part of the scenery in the garden can be leaked out through ornamental perforated windows for pedestrians to see, so as to arouse people's interest in visiting the garden. Ornamental perforated windows have the functions of ventilating and lighting, exposing and separating the scenery, and beautifying the walls. Ornamental perforated windows are set up on the walls and corridors with scenery on both sides, which can be used to obstruct the scenery as well as allow people to see the scenery inside and outside the garden. In addition, the ornamental perforated window itself is exquisite in shape, just like the exquisite threedimensional patterns on the white wall, breaking the dull atmosphere of the wall, and adding a bit of lively interest to the garden. The interwalls in the garden are often built with windows to leak the scenery, making visitors feel overwhelmed.

4) The environment emerges from the scene: There are a lot of poetic and pictorial splendor related to the white walls and ornamental perforated windows of the garden. It combines with other buildings through walls, and forms a unique scene with appropriate plant furnishing and stone peaks, water light and tree shadows. This kind of distant courtyard deep feeling and the hazy looming beauty of the scene is revealed by this scene. A small space enclosed by a wall can give people a sense of security and seclusion. At the same time, the birds' twitter and fragrance of flowers, bamboo shadows, chirping of cicadas, and water sounds outside the wall or courtyard will create a sense of novelty. These are all suitable to set off the artistic conception.

\section{B. Configuration with other garden elements}

1) Garden walls and other buildings: When dividing the space in the garden, if the wall is merely used to separate the space, it is tedious. Therefore, the garden wall is often combined with other buildings to form garden spaces of different forms. The garden wall is combined with the house building to form a Qingzhai (a quiet room), a mansion with many courtyards and very high walls, a garden, a spacious courtyard and other spaces. The combination of garden wall and pavilion can form a pond waterscape; the combination of garden wall and veranda can form a closed space; the combination of the garden wall and the garden road can form rain channels, mountain paths leading to seclusion, and an alley sandwiched on both sides of the street and multiple channels. The relationship between the wall and the door is very close.The garden wall and garden gate constitute the main entrance and exit, which are the face of the garden and must be designed carefully. Earthen walls should be matched with wooden gates, brick walls with festoon gate, palace walls with palace gates or temple gates, and white walls with painted doors. To show the natural, graceful and poised style of the garden, the door wall is often spread out with out-toe pattern outside, forming a gate and courtyard with eaves gallery, steps or stone lions. On the wall of the inner garden, there are more round gates or other style gates to show uniqueness. When it is necessary to borrow and contrast the scenery, the gate is often opened facing each other, or covered by artificial hills, screens, and screen walls facing the gate of a house.

2) Garden walls and hillstones and water bodies: Using the smooth texture of the walls to set off other garden elements is a common method in Classical Gardens of Suzhou. The garden wall is often staggered with the texture of the rock. The climbing wall is stacked up and down to enrich the change of the wall, or the cross-section of the climbing wall is used to enrich the line and surface form of the plan composition. In order to make the landscaping lively, local rocks can also be introduced into the garden through the wall to increase the changing taste of the elevation wall plane land. The combination of wall and water is very common in gardens. Water is flowing, invisible, and transparent. And walls are hard, tangible, 
and heavy. The difference in texture between the two makes them complement each other and bring out the best in each other. When the garden wall meets the water surface, it can be transitioned by a bridge. The garden wall built along the fair current should be appropriately twisted to echo the movement of the water body and be set off by bamboo.

3) Garden walls and plant furnishing: Walls in gardens often appear as the background of plants. In fact, the relationship between walls and plants at this time is mutually dependent. The plant furnishing of the inner wall of the garden needs to be based on the needs of landscaping, and the plant furnishing of the outer wall of the garden is often a part of the whole garden landscape, and it is also the need for the beautification of the garden wall itself. When arranging plants, it is necessary to take into account the needs of the overall layout and grouping of scenery, highlight the characteristics of the garden according to the type, nature, and characteristics of the garden, and create the artistic conception of the garden. The main purpose of arranging plants is first to beautify the garden wall itself; the second is to assist the performance of the characteristics of the garden; the third is to reveal the best local scenery and shield the places that should not be leaked; the fourth is to communicate the space inside and outside the wall through flowers, trees, and bamboo, and trees to enrich the landscape level. The arrangement of plants is an artistic choice. Plants must be arranged sparsely and densely, with different heights and different varieties, and evergreen and deciduous plants should also be arranged together to reflect the artistic conception of landscaping. The focus of the garden wall plant configuration is the garden gate area. Generally, earthen walls, white walls, and brick walls are often equipped with fernleaf hedge bamboo, nandina, peach trees, plum trees, apricot trees, pomegranate trees, Syzygium aromaticum, Cryptomeria fortunei, Chinese parasol, etc.

\section{Unique artistic conception expression}

1) Wall balance: According to the advanced view of nature, traditional gardens must make every effort to harmonize the naturalness of the garden with the form of the structure when constructing the garden to find the balance between art and technology, form and content, and man and nature. However, the establishment of the wall seems to be somewhat contradictory to the open state that requires people to approach nature in the conception of nature. But since it is a functional requirement, there is a necessity for its existence. Traditional theoretical explanations and actual practices aim to utilize, coordinate and balance this contradiction.
On the one hand, it emphasizes the necessity of the existence of the wall, because it is conducive to regional security and definition, and has a protective function in a specific stage of social development; on the other hand, it is necessary to try to weaken the protection and distinction function of the wall. The wall becomes the intermediary part of constructing the garden, which not only has the function of protection and distinction, but also the function of framing scenery and viewing scenery. This choice of "likeness and dislikeness" gives the walls of traditional gardens the attribute of "balance". Classical Gardens of Suzhou use compromise techniques to maximize the balance of the walls, turning disadvantages into advantages, and forming a unique style.

The wall construction is natural, and the material selection and form design are of very important significance. As the saying goes, "People in the world building walls lack ingenious ideas and handling methods because of irregular foundations and arbitrary construction. Why can't the wall be built with one end high and wide, and one end low and narrow, in accordance with the regular and straight form of the house? Ordinary craftsmen can't understand this ingenious design concept". It shows that the wall can't be treated in isolation, but should be integrated with the garden building and treated as a combined element to create a garden landscape effect. The full text reveals the rules of building a garden based on the natural approach, and indirectly indicates the balance of the walls and the compromise treatment based on this.

2) The poetic and pictorial splendor of walls: The art of gardening has a profound relationship with literature and painting art. In particular, influenced by the freehand landscape paintings of literati in the Tang and Song dynasties, the art of gardening shrinked the natural landscape and reproduced it on paper, and the garden maker created the exquisite pictures on the paper in front of the white walls of Classical Gardens of Suzhou. The expression of Classical Gardens of Suzhou art tends to "be implicit beauty", "hide and reveal the open and deep of the famous mountains and wonders", and "have dense willow trees and bright flowers", which stems from the unique and long-standing cultural tradition and aesthetic interest of this nation. This feature is exactly the same as that of Chinese classical poetry and painting. Traditional gardening art often avoids shallow exposure and pursues the depth of artistic conception. The garden space emphasizes that "It will be too deep if it's separated, and it will be too shallow if it's broken through". Garden builders often use the technique of compliment after criticism, and hiding first if it wants to present, "hiding" the real beautiful scenery. In the carefully prepared "hiding", 
there is also "presenting", making it looming and intangible. The ingenuity of "hiding" and "presenting" is inseparable from the garden wall, and the poetic sentiment expressed by the garden wall in Classical Gardens of Suzhou is also here.

The encounter of environment and mind is a major feature of the Chinese Classical Gardens of Suzhou built by literati, which emphasizes the perfect fit between the external objects and the inner ideal world. A flower, a tree, and a stone can trigger a lively world of artistic conception. As one of the elements of gardening, the wall will inevitably be affected by this kind of thinking, so the wall in the classical garden forms a unique artistic conception.

\section{Conclusion}

The wall is the most basic and most important physical element in traditional Suzhou buildings. Utilizing the attributes and shaping functions of walls in traditional Suzhou buildings, through the changes in the form of the wall space, the changes in the combination and configuration relationship between the walls, and between the walls and other spatial entity elements, different landscaping techniques are shown to create Suzhou traditional architectural spaces with different personalities.

\section{References}

[1] Yang Shu. Research on the Wall of Traditional Buildings in Suzhou [D]. Suzhou University, 2010 (7). (in Chinese)

[2] Guo Rongrong. The Wall in the Garden [D]. Beijing Forestry University, 2009. (in Chinese)

[3] Ni Suning. On the Artistic Characteristics of Suzhou Garden Space [D]. Suzhou University, 2002. (in Chinese)

[4] Sun Tao. The Role of Walls in Framing [J]. Shanxi Architecture, 2007(33). (in Chinese)

[5] Cao Lindi. Chinese Garden Culture [M]. China Building Industry Press, 2005. (in Chinese)

[6] Peng Yigang. Analysis of Chinese Classical Gardens [M]. Beijing: China Building Industry Press, 1986. (in Chinese)

[7] Yin Wen. Talking About the Wall [M]. Shandong: Shandong Pictorial Publishing House, 2005. (in Chinese)

[8] Tong Jun. Journal of Jiangnan Gardens [M]. Beijing: China Building Industry Press, 2009. (in Chinese)

[9] Chen Congzhou. Dictionary of Chinese Garden Appreciation [M], Shanghai: East China Normal University Press, 2001. (in Chinese)

[10] Liu Dunzhen. Classical Gardens of Suzhou [M]. Beijing: China Building Industry Press, 1979. (in Chinese)

[11] Liang Dunmu. Trying to Talk About the Garden Wall [J]. Guangzhou Gardens, 1995 (2). (in Chinese)

[12] Sun Tao. The Role of Walls in Framing [J]. Shanxi Architecture, 2007 (11). (in Chinese) 